well as to define characteristics of the lymphoma and its evolution.

Methods Retrospective observational, longitudinal study conducted in a tertiary hospital. Medical records of 362 patients with $\geq 4$ SLICC classification criteria of SLE were reviewed, including those with lymphoma diagnosis. Demographic and clinical data, comorbidities, SLE manifestations and therapy, data related to lymphoma and outcome were collected. Descriptive statistic analysis with measures of central tendency and measures of variability was performed.

Results Of the 362 SLE patients, 9 (2.5\%) were diagnosed of lymphoma, of which $100 \%$ female. Mean age at SLE diagnosis was 34 y.o (SD 11) and average duration from SLE diagnosis to lymphoma was 17 years (SD 14). 7 patients were Caucasian and 2 Hispanic. Observed comorbidities were hypertension (67\%), diabetes (22\%), dyslipidemia (33\%), HBV infection (11\%) and active smoking (66\%). No malignancy history was detected. Most frequent SLE features were haematological (100\%), joint (56\%) and skin (56\%) involvement. The serious ones were: 3 patients with haemolytic anaemia (1 of them, platelets <20000), 2 epilepsia (1 with CNS vasculitis), 1 glomerulonephritis, 1 pulmonary hypertension and 1 hemophagocytic syndrome. Only 1 patient had overlap with Sjögren's syndrome. At the time of lymphoma diagnosis, 7 patients were on steroids, 4 on immunosuppressants (2 mycophenolate, 1 azathioprine, 1 rituximab) and 3 on antimalarials (table 1). Mean age at lymphoma diagnosis was 51 y.o (SD 10). 5 patients $(56 \%)$ had diffuse large B-cell lymphoma (DLBCL); 1, NHL; 1, Hodgkin's lymphoma; 1, mantle B-cell lymphoma and; 1, MALT. Only 1 patient, of 4 with available data, had EBV positive in the tissue. 7 patients received chemotherapy and 2 patients completed treatment with autologous peripheral stem-cell transplantation. Three patients died, 2 due to lymphoma and one due to other causes (severe flaccid paralysis). Overall survival after lymphoma diagnosis was 8 years (SD 6).

Conclusion In our patients, unlike that reported in the literature, lymphoma diagnosis was in SLE with longer duration of the disease, and all cases were female. Most frequent subtype was NHL, and all patients had previous haematological manifestations. Regarding previous SLE treatments, 5 patients had been exposed to immunosuppressants.

\section{P166 POSSIBLE NEW ROLE FOR HCQ IN PREVENTING DEPRESSION IN JSLE?}

${ }^{1}$ Sara Ganhão, ${ }^{2}$ Beatriz Silva, ${ }^{1}$ Francisca Aguiar, ${ }^{1}$ Mariana Rodrigues, 1,2Iva Brito, 2,3 Margarida Figueiredo-Braga. 'Young Adult and Pediatric Rheumatology Unit, Centro Hospitalar e Universitário do Hospital de São João, Porto; ${ }^{2}$ Faculty of Medicine, University of Porto, Porto; ${ }^{3}$ Dept. of Clinical Neurosciences and Mental Health, Porto, Portugal

\subsection{6/lupus-2020-eurolupus.208}

Background Hydroxychloroquine (HCQ) is a key immunomodulatory treatment in systemic lupus erythematosus (SLE) with pleiotropic effects. Beyond anti-thrombotic, anti-atherosclerotic and anti-diabetic effects, anti-microbial and anti-cancer are possible roles. Neuropsychiatric symptoms, mostly headaches, depression, anxiety and cognitive impairment, affect nearly half of patients. Several pathways have been identified: antibody-mediated/cytokine-induced neurotoxicity, vasculopathy and loss of neuroplasticity. Thus, we hypothesized if there is a role for HCQ in preventing depression in jSLE.
Methods A cross-sectional sample of juvenile-onset SLE (jSLE) patients, currently aged $\geq 16$ years, completed a psychosocial assessment including the SF-36, HADS, SHS, BriefCope and MMSE questionnaires, between October 2018- May 2019. Local Ethics Committee approved the study. All patients fulfilled both 2012 and 2019 EULAR/ACR classification criteria for SLE. Juvenile-onset was defined as age at diagnosis $<18$ years. Demographics and clinical characteristics were collected. Statistical analysis was performed with SPSS ${ }^{\circledR}$.

Results 30 jSLE patients were included (90\%female) in the study, with median age of 21 years, being the youngest 16 and the oldest 35 , with mean (SD) age of diagnosis of 15.8 \pm 2.1. Mean values (SD) of psychosocial assessment were: SHS 5.2 (1.02); MMSE of 27.7 (1.8); Physical health SF-36 of 66.8 (9.9) and Mental health SF-36 of 68.9 (17.5). 23.3\% jSLE showed mild cognitive impairment, 63.3\% anxiety and $13.3 \%$ depression. From the 27 jSLE treated with HCQ, those had better results in the SHS $(p=0.030)$ and scored lower in scores in the Hospital Anxiety and Depression scale $(p=0.023)$. Interestingly, this also occurs for emotion focused coping, with significantly better results in jSLE taking HCQ $(\mathrm{p}=0.001)$.

Conclusions Young adults with SLE are at risk for depression and HCQ may have a role in preventing it. Longitudinal studies will permit to confirm present results and clarify the role of coping strategies in the occurrence of depression in jSLE.

\section{P167 LONG TERM FOLLOW-UP OF LUPUS PATIENTS UNDER ANTIMALARIC TREATMENT: FACTORS OF DROP-OUT}

Roxana González-Mazarío, Jorge Juan Fragío Gil, José Ivorra Cortés, Elena Grau-García, Luis González-Puig, Francisco Miguel Ortiz Sanjuan, Samuel Leal-Rodriguez, Isabel MartínezCordellat, Rosa Negueroles-Albuixech, José Eloy Oller-Rodríguez, Marta De-la-RubiaNavarro, Inmaculada Chalmeta-Verdejo, Cristina Alcañiz-Escandell, Cristóbal Pavez-Perales, Elvira Vicens-Bernabeu, Carmen Nájera-Herranz, Inés Cánovas-Olmos, José Andrés RománIvorra. Rheumatology Dept., Hospital La Fe, Valencia, Spain

\subsection{6/lupus-2020-eurolupus.209}

Background Antimalarials represent the cornerstone of SLE treatment, since its uses control clinical manifestations in many patients, prevents disease flare and permits steroid reduction. The aim of this study is to describe the safety profile and the reasons for discontinuation of antimalarials in patients with SLE and determine which factors act as a predictor of drop-out.

Methods A single centre, retrospective, case control study was performed including patients with SLE according to SLICC 2012 criteria. Clinical and demographical variables were collected. Disease activity was measured with clinical, analytical and disease scores.

Results 66 patients were included, 56 patients (84.8\%) were females, the median age was 49.3 years $(23.4,76.2)$. $95.50 \%$ of patients were Caucasian. 11 patients (16.7\%) had high blood pressure and $6(9.1 \%)$ diabetes mellitus. The disease duration of SLE had a median of 198 months (5.1, 144.9), and median SLEDAI was 3.4 (2-23). 45 patients (68.2\%) were taking steroids and its median dosage was $3,6(1.2,2.5)$ mg. 58 patients received antimalarial treatment during their follow-up with a median exposure time of $354(6,867)$ months. 91.2\% took hydroxychloroquine (HCQ), 6.9\% chloroquine (CQ), and only one patient mepacrine (1.7\%). At least one side effect was reported in 22 patients $(33.3 \%)$ leading to permanent withdrawal in $13(19.7 \%)$ : 7 cases of ocular toxicity, 4 intolerance $(6,1 \%)$, and 2 cases of inefficacy (3\%). 45 